\title{
Comparison between the electrokinetic properties of kaolinite and montmorillonite suspensions at different volume fractions
}

\author{
Y.Tsujimoto ${ }^{1}$, C. Chassagne ${ }^{2, *}$, Y.Adachi ${ }^{1}$ \\ ${ }^{1}$ Graduate School of Life and Environmental Sciences, University of Tsukuba,
}

1-1-1, Tennnoudai, Tsukuba-shi, Ibaraki, 305-8572, Japan

${ }^{2}$ Department of Environmental Fluid Mechanics, Faculty of Civil Engineering and Geosciences, Delft

University of Technology, Box 5048, 2600 GA, The Netherlands

*Corresponding author.

E-mail address: C.Chassagne@tudelft.nl 


\begin{abstract}
We have investigated the electrokinetic responses of two different kinds of clay particles, kaolinite and montmorillonite. The dielectric permittivity of kaolinite suspensions is linearly proportional to volume fraction up to volume fractions of $20 \%$, whereas that of montmorillonite is deviating from a linear relationship, for volume fractions below $0.5 \%$. This indicates that the montmorillonite particles experience a particle-particle interaction at these low volume fractions. The complex dipole coefficients of both clays estimated by experimental data are however within experimental error in good approximation independent on volume fraction and agree with the theoretical predictions. The relaxation frequency in clay-water system at low ionic strength is almost determined by the relaxation of the double layer for both kaolinite and montmorillonite. For volume fractions larger than $0.5 \%$ for montmorillonite we find that the zeta potential measured by electroacoustic methods starts to depend strongly on volume fraction. It is expected that for these high volume fractions the dipole coefficients will also become volume-fraction dependent.
\end{abstract}

Key words: Dielectric spectroscopy, montmotillonite, kaolinite, dipole coefficient 


\section{Introduction}

The permittivity and conductivity of clay suspensions have been investigated by numerous authors [1-14]. In a previous article [15], we have investigated both the electrophoretic mobility and dielectric spectroscopy response of dilute montmorillonite particle suspensions of montmorillonite $(0.1 \%$ volume fraction). We have found values comparable to what other authors find for this system, using the same techniques. However, we also noted a deviation from our results compared to those of Rasmusson et al. [5], who similarly studied dielectric spectroscopy and electrophoretic mobility responses. These authors used a volume fraction of 0.4 or $0.8 \%$ for the size and mobility measurements where they used a AcoustoSizer from Matec Applied Sciences. On the other hand, for the complex conductivity measurements (corresponding to our dielectric spectroscopy measurements), they used 2 or $4 \%$ volume fraction [5]. The authors found that the electroacoustic signal (ESA) used to assess the dynamic mobility of the particles was independent of the stirrer speed, implying that the particles were randomly oriented in this experiment. For the lower volume fractions we used in the dynamic mobility experiments $(0.025 \%, 0.05 \%$ and $0.1 \%)$, there were similarly no changes in electrokinetic response. The question remains however how particles behave during a dielectric spectroscopy (complex conductivity) experiment, where the suspension is not stirred, and the volume fraction 10 times higher.

In this article, we would like to investigate into more detail the dependence of the complex conductivity response as a function of volume fraction. We do this for two types of clays: kaolinite and montmorillonite. In order to get the best information about particle interaction, we favored to study a system with as low ionic strength as possible. The Debye length $\kappa^{-1}$ around the charged particles is then very large, and particle-particle interactions are more likely to occur.

We are going to compare the results we obtain with the results obtained by Rasmusson et al. and other authors that work at different volume fractions.

\section{Experimental}

\subsection{Sample preparation}

The kaolinite (China clay) was obtained from VE-KA Industry Keramische Grondstoffen Ltd., The 
Netherlands. The average size of the primary particle was found to be around $10 \mu \mathrm{m}$ [16]. This clay sample was prepared by dispersing the powder as received in millipore water. The suspension was ultrasonic shaken for 5 minutes prior use.

Montmorillonite were obtained from commercially available Kunipia- F (Kunimine Industry Co. Ltd.). Coarse components, mainly silica sands, contained in the purchased material were removed by sedimentation. To replace the surface cations by sodium ions, we dispersed the clay particles into the 2.0 M NaCl solution for weeks and occasionally replaced the clear supernatant by a new $2.0 \mathrm{M} \mathrm{NaCl}$ solution until the substitution was completed. Salt free stock dispersions were prepared by repeatedly dialyzing the suspension against distilled water until the electric conductivity became less than 1.5 $\mu \mathrm{S} / \mathrm{cm}$. After these treatments, this suspension was stocked after freeze-drying. Dielectric spectroscopy measurements were performed with the suspensions made from the stock freeze-dried montmorillonite diluted with an aqueous solution of sodium chloride of known concentration. The value of $\mathrm{pH}$ in the suspension was not purposely controlled, but was measured to be between 5.5 and 6.5 throughout all the experiments. As an equivalent diameter, we measured $382 \mathrm{~nm}$ by dynamic light scattering measurements.

\subsection{Dielectric Spectroscopy}

The details of this measurement technique, and the theory can be found in [15]. The electrokinetic response of the clay suspensions were measured in the range $10^{4} \mathrm{~Hz}-1.5 \times 10^{7} \mathrm{~Hz}$ using a homemade concentric cylindrical measurement cell [17]. The impedance $Z$ measured by the dielectric spectroscopy device can be seen as the impedance of an equivalent circuit formed by a resistance $R$ and conductance $C$ in parallel. Therefore:

$$
\frac{1}{Z}=\frac{1}{R}+i C \omega
$$

From the measured $\mathrm{R}$ and $\mathrm{C}$, the conductivity and permittivity are calculated according to the following formula [18];

$$
\mathrm{K}=\frac{1}{2 \pi L} \operatorname{Re}\left(\frac{1}{Z}\right)
$$




$$
\varepsilon=\frac{1}{2 \pi \varepsilon_{0} L \omega} \operatorname{Im}\left(\frac{1}{Z}\right)
$$

$L$ represents the height of liquid in the cell. This cell was filled at start with $45 \mathrm{~mL}$ suspension. Measurements for one sample repeated four times by removing $10 \mathrm{~mL}$ from the suspension. The conductivity and permittivity were estimated from the slope of the variation of $1 / \mathrm{Z}$ as function of the variation of liquid level. "Blank" solutions i.e. electrolyte solutions of same conductivity as the suspensions were also measured. The conductivity and permittivity of the particles were calculated by subtracting the blank solution response from the conductivity and permittivity responses of the suspensions. Doing so, the effect of electrode polarization was minimized [18]. All measurements were performed at $25^{\circ} \mathrm{C}$.

Eq.(1) of Ref.[15]:

$$
\widetilde{\mathrm{K}}=\widetilde{K_{1}}(1+3 \varnothing \beta)
$$

where $\bar{K}=K+i \omega \varepsilon_{0} \varepsilon$, where $K$ is the conductivity, $\omega$ is the frequency (rad/s), $i$ is the square root of -1 , $\varepsilon$ is the relative dielectric permittivity and $\varepsilon_{0}$ is the dielectric permittivity of vacuum. $K_{1}, \varphi$ and $\beta$ indicate the conductivity of electrolyte, volume fraction and dipole coefficient, respectively. This equation was derived under the assumption that there is no particle-particle interaction. From this equation, we obtain:

$$
\begin{aligned}
& \Delta \varepsilon \equiv \frac{\varepsilon-\varepsilon_{1}}{\emptyset}=3\left[\operatorname{Re}(\beta)+K_{1} \frac{\operatorname{Im}(\beta)}{\omega \varepsilon_{0}}\right] \\
& \Delta \mathrm{K} \equiv \frac{K-K_{1}}{\emptyset}=3\left[K_{1} \operatorname{Re}(\beta)-\varepsilon_{0} \varepsilon_{1} \operatorname{Im}(\beta)\right]
\end{aligned}
$$

where $\varepsilon_{1}$ indicates the dielectric permittivity of the electrolyte. The dipole coefficient is then found from the measured conductivity and permittivity by inverting these equations.

$\beta$ is dependent on two contributions, $\beta_{n}$ and $\beta_{p}$ which represents the dipole coefficient in the direction perpendicular to the electric field or along it, respectively. In case that (as assumed here) the electric field is weak enough, we have [15]:

$$
\beta=\frac{1}{3} \beta_{p}+\frac{2}{3} \beta_{n}
$$

\subsection{Electrophoresis}

The electrophoretic mobilities $U_{s}$ were measured at low frequency by Doppler velocimetry using a 
Malvern Zetasizer Zeta-Nano. The electrophoretic mobility is plotted for convenience of units using the

Smoluchowski formula

$$
\mathrm{U}_{s}=\frac{\varepsilon_{0} \varepsilon_{r} \zeta}{\eta}
$$

which gives a linear relationship between mobility and zeta potential $\zeta$, given the dielectric permittivity and the viscosity. We use: $\varepsilon_{r}=78.55$ and $\eta=0.89 \mathrm{mPa}$.

At high frequency the suspension's electroacoustic response was measured at $3.3 \mathrm{MHz}$ using the Dispersion Technology device DT-300. The zeta potential obtained from the electroacoustic device was given by the manufacturer [19].

\section{Results of Dielectric Spectroscopy}

\subsection{Kaolinite suspensions}

\subsection{1 conductivity and dielectric increment}

The conductivity and dielectric permittivity of the studied kaolinite suspensions is shown in Fig.1a and $1 \mathrm{~b}$ as function of volume fraction, for several applied field frequencies. We observe that the conductivity is linear with volume fractions above 9.2\%. The dash line in Fig.1a is meant as a guide for the eye. Under $9.2 \%$ volume fraction the conductivity is almost constant and roughly equal to $0.024 \mathrm{~S} / \mathrm{m}$. This conductivity value corresponds to ionic strength of $1.4 \mathrm{mM}$ of $\mathrm{KCl}$, which is quite large, considering the fact that no salt has been added to the suspension. This conductivity derives from the fact that the kaolinite has not been properly cleaned before use, so some ions release from the clay to form a background electrolyte concentration. The linearity between both conductivity and permittivity upon volume fraction indicates that particle-particle interaction can be neglected for kaolinite in the volume fraction range [0\% - 20\%].

Raythatha et al. [6] studied the high-frequency dielectric response of kaolinite (in the MHz regime). They report that the kaolinite sample they study give $\varepsilon-\varepsilon_{1}=0$ for the whole frequency range, below a volume fraction of $9.6 \%$. At $9.6 \%$ volume fraction, they find that $\varepsilon-\varepsilon_{1}=25$ at $0.5 \mathrm{MHz}$. This is also in line with our results at $0.5 \mathrm{MHz}$, as, we find $\varepsilon-\varepsilon_{1}=29$ for $14 \%$ volume fraction. For lower volume fractions we find $\varepsilon-\varepsilon_{1}=9$ (4\%), $\varepsilon-\varepsilon_{1}=11$ (7\%) and $\varepsilon-\varepsilon_{1}=16$ (9\%). For any 
frequency the dielectric permittivity is almost linear with volume fraction (see Fig.1b), indicating that there is no particle-particle interaction for the low volume fractions.

In order to better study the relation between surface properties of kaolinite and its dielectric response, we will analyze the data in terms of dipole coefficients. These (complex) dipole coefficients are obtained from eq.(4). Note that the dipole coefficient $\beta$ is in principle independent on volume fraction when $\varepsilon-\varepsilon_{1}$ is linearly dependent on volume fraction.

\subsection{2 dipole coefficient}

In Fig.2, we plotted the real and imaginary parts of the dipole coefficient as a function of the frequency for 5 volume fractions. The dipole coefficient for 20.8, 14.4 and $4.6 \%$ volume fraction are similar, whereas the dipole coefficients at volume fraction $9.2 \%$ and $7.4 \%$ are slightly lower. These discrepancies were already visible in the conductivity measurements results. They probably arise from experimental errors. As can be verified from eqs.(4), at low frequency, we have:

$$
\begin{aligned}
& \operatorname{Re}(\beta) \approx \Delta K / 3 K_{1} \\
& \operatorname{Im}(\beta) \approx \Delta \varepsilon \omega \varepsilon_{0} / 3 K_{1}
\end{aligned}
$$

For all measurements, the imaginary part of $\beta$ is almost zero at low frequencies. This is expected, since, as can be seen from eq.(6), $\Delta \varepsilon$ is a finite number, $\operatorname{Im}(\beta)$ has to be very small at low frequency. A remarkable feature of $\operatorname{Im}(\beta)$ is that all curves cross at about $0.1 \mathrm{MHz}$ for any volume fraction. This feature was also found in the plot of the permittivity of suspensions as function of frequency but for a slightly higher frequency.

Ishida et al. investigated the dielectric relaxation of kaolinite particles of size $<2 \mu \mathrm{m}$ in aqueous media in the frequency range $10^{3} \mathrm{~Hz}$ to $10^{10} \mathrm{~Hz}$ [9]. The volume fraction of kaolinite suspensions they used was $50.6 \%$, much higher than what we used. From their absorption curves, we could deduce that the suspensions had a conductivity of about $10^{-4} \mathrm{~S} / \mathrm{m}$, which is much lower than our conductivity, as their suspensions were thoroughly purified. The $\mathrm{pH}$ of the suspension was 9. From the analysis of their curves, they discovered three relaxation frequencies. The highest relaxation frequency (at $20 \mathrm{GHz}$ ) corresponds to the relaxation of bulk water molecules. The relaxation frequency near $50 \mathrm{MHz}$ is found 
to be due to the relaxation of water molecules bound to the clay surface. The last relaxation frequency around $3 \times 10^{5} \mathrm{~Hz}$ is due to the polarization of the system composed by the clay particle and its ionic double layer. In our measurements, only this last frequency is assessable, since we studied the system between $10^{4}$ and $1.5 \times 10^{7} \mathrm{~Hz}$. We found (see Fig.2) that the relaxation frequency of kaolinite is around $5 \mathrm{MHz}$ regardless of volume fraction. The fact that our relaxation frequency is larger than the one observed by Ishida et al. is most probably due to the background electrolyte concentration, since the relaxation frequency is known to shift to higher frequencies for larger ionic strengths [15].

In Fig.3 we attempt to fit the theoretical dipole coefficient according to the procedure described in Ref.[15]. We used the same zeta potential (-75 mV) and almost the same Stern layer conductance (0.0205 S/m instead of $0.0180 \mathrm{~S} / \mathrm{m})$ as we did in the fit of similar kaolinite suspensions [20]. The relaxation frequency can be estimated roughly from $D \kappa^{2}$ (where $D$ is the diffusion coefficient of ions) which is of order of $4 \mathrm{MHz}$. The same relaxation frequency was found in [20], where the kaolinite was dispersed in an $1.5 \mathrm{mM}$ of $\mathrm{KCl}$ electrolyte solution. This agrees well with the fact that the background electrolyte concentration in the present study is around $1.4 \mathrm{mM}$ of added monovalent salt.

The dipole coefficient for $9.2 \%$ and $7.4 \%$ suspensions are more in line with the predicted value for $\beta_{n}$. It would be tempting to say that particles at these volume fractions are aligned with their n-axis in the direction of the electric field. However, the difference between $\beta_{n}$ and $\beta$ is within the experimental error, and since the value for the $4.6 \%$ suspension is almost identical to the $20.8 \%$ suspension this support the fact that the dipole coefficient do not depend on volume fraction in the volume fraction range investigated.

We observe that it is impossible to fit the low-frequency amplitude of the dielectric response without a Stern layer. The same conclusion was drawn by Rowlands and O’Brien [3] who also studied the dielectric response of kaolinite, but at high frequency $(1-40 \mathrm{MHz})$. They use a volume fraction of $1.6 \%$ volume fraction, at $\mathrm{pH}=7-8$ and several added salt concentration, from approx. $1 \mathrm{mM}$ to $10 \mathrm{mM}$. The curves they obtained as function of frequency for different ionic strength also cross, as our do, but at different frequencies (10, 20 and $30 \mathrm{MHz})$. The theoretical curves for $\operatorname{Im}(\beta)$ without Stern layer conductance do never cross at any measured frequency when the ionic strength is varied. When a Stern 
layer conductance is used (the same for all ionic strengths), the curves all cross at the same frequency, in line with what happens for our experimental $\operatorname{Im}(\beta)$ for different volume fractions. Rowlands and O’Brien had to use different Stern layer conductances to fit their data which might be the reason that their measured cross-over frequencies are different, as the value of the theoretical cross-over frequency depends on the value of the Stern layer conductance. A more detailed study (varying systematically both ionic strength and volume fraction) would be required in order to investigate the relation between Stern layer conductance and relaxation frequency.

\subsection{Montmorillonite suspensions}

\subsection{1 conductivity and dielectric increment}

The conductivity and dielectric permittivity of montmorillonite suspensions are shown in Fig. 4a and b.

The conductivity is linear with volume fraction The electrophoretic mobility of both ions and montmorillonite particles is therefore not significantly hindered by the presence of montmorillonite particles. Extrapolating the conductivity at low volume fractions we find a ionic conductivity (no particles) of $K_{1}=2 \times 10^{-4} \mathrm{~S} / \mathrm{m}$ which is corresponding to $0.015 \mathrm{mM}$ of added $\mathrm{NaCl}$. This value is very low, and even though we do not know the type of ions present in the electrolyte, we believe that a large part could be $\mathrm{Na}^{+}$and $\mathrm{Cl}^{-}$, since the montmorillonite has been saturated by $\mathrm{NaCl}$. Raythatha et al [6] did not find a linear dependence for the conductivity of their suspension of Na-montmorillonite in distilled water. They assumed this was due to the fact that their sample (3\% volume fraction) was a thick paste and ions mobility was hindered.

Raythatha et al. [6] have investigated the high-frequency $(0.5 \mathrm{MHz}-1.3 \mathrm{GHz})$ response of montmorillonite particles in distilled water as a function of volume fraction. They found, as we did, a strong dependence on the permittivity on volume fraction. In their case they found for $0.5 \mathrm{MHz}, \varepsilon-\varepsilon_{1}$ $=0\left(0.004 \%\right.$ volume fraction), $\varepsilon-\varepsilon_{1}=115(0.5 \%)$ and $\varepsilon-\varepsilon_{1}=200(1 \%)$. From our results, we find the relation $\varepsilon-\varepsilon_{1}=-2+433 \varphi-192 \varphi^{2}$ where $\varphi=100 \phi$ where $\phi$ is the volume fraction. It can be verified that the values found by Raythatha are in good agreement with the ones obtained from this relation. 
We consider that the dependence of dielectric permittivity on volume fraction is the result of the long-range interactions between particles. In previous investigations, Adachi et al. [21] and Tsujimoto et al [22] measured the relative viscosity of montmorillonite suspensions under dispersed conditions to investigate the electroviscous effect. They found that at especially salt-free conditions, the long-range interaction is remarkable. They also showed that even if the distance between particles is larger than the Debye length (which is certainly the case for 2 or $2.5 \mathrm{mM}$ of $\mathrm{NaCl}$ and the volume fraction used), these interactions are significant. Arroyo at a. [23] studied the electrokinetic properties of montmorillonite as function of $\mathrm{pH}$, at $0.1 \mathrm{mM}$ of added $\mathrm{NaCl}$ and found almost the same dielectric permittivity increment as we do for $\mathrm{pH}=5$ or 7 (the curves superposed for these two $\mathrm{pH}$ ), for $0.2 \%$ volume fraction. They verified that at $\mathrm{pH}=9$, the dielectric increment was linearly proportional to the volume fraction, implying that no particle-particle is present. At lower $\mathrm{pH}$, they found, like we do, a deviation from linearity which is related to the interaction between (slightly) positive edges and negatively charged faces.

\subsection{2 dipole coefficient}

The dipole coefficients derived from the measurements are displayed in Fig.5. We observe that the low-frequency amplitude of $\operatorname{Re}(\beta)$ is $500 \sim 1000$ times higher than the amplitude of the kaolinite suspensions. This is due to the fact the ionic strength in the system is so low and the particles so small (small ка), as compared to the kaolinite samples.

Ishida et al. investigated the dielectric relaxation of montmorillonite particles in aqueous media in the frequency range $10^{3} \mathrm{~Hz}$ to $10^{10} \mathrm{~Hz}$ [9]. The volume fraction of montmorillonite suspensions they used was $5 \%$, much higher than what we used; the conductivity was probably close to the one of their kaolinite sample, but the absorption curve was not given. The $\mathrm{pH}$ of the suspension was 9. Like what they found for kaolinite, the same three relaxation frequencies were observed. In particular, they also find a relaxation frequency near $100 \mathrm{MHz}$ which they attribute to the relaxation of water molecules bound to the clay surface. They find a lower relaxation frequency at about 3.8 MHz which is higher than 
the relaxation frequency observed in Fig.5, which is probably due to the higher ionic strength of Ishida's samples.

As we did in [15] and for kaolinite, we fitted the theoretical prediction to the experimental dipole coefficient as shown in Fig.6. We used a zeta potential of $-70 \mathrm{mV}$ and a Stern layer conductance of $0.044 \mathrm{~S} / \mathrm{m}$. The value of the zeta potential however is barely affecting the fit and could be taken in the range $[-60 \mathrm{mV} ;-80 \mathrm{mV}]$ for it changed the values of only a few percent. In order to correctly estimate the relaxation frequency at around $10^{5} \mathrm{~Hz}$ when we use $0.032 \mathrm{mM}$ as the value of ionic strength, corresponding to $K_{1}=4.9 \times 10^{-4} \mathrm{~S} / \mathrm{m}$.

\section{Results of electrophoretic mobility}

In Fig. 7 we show the obtained zeta potential as function of volume fraction for both kaolinite and montmorillonite. Note that is was impossible to measure the kaolinite suspensions with the ZetaNano as the suspensions were too turbid at the volume fractions used.

\subsection{Kaolinite suspensions}

The three values obtained for kaolinite do not display a trend. The average zeta potential found by the DT-300 is about $-30 \mathrm{mV}$. When a volume fraction of $8 \%$ was used, we got a zeta potential of $+8 \mathrm{mV}$.

Rowlands et al. investigated both the dielectric and electrophoretic response of kaolinite particles [3], [4]. They measured the high-frequency electrophoretic and dielectric response of the suspensions for several ionic strengths at a volume fraction of $1.6 \%$. Their results for the dipole moment are discussed above. As for the electrophoretic mobility, they find a zeta potential of - $58 \mathrm{mV}$ when the mobility is converted into zeta potential using Smoluchowski formula, for their lowest ionic strength used (1.3 $\mathrm{mM})$. Since no clear trend could be obtained from our measurements, it is impossible to comment what the expected zeta potential would be at $1.6 \%$ volume fraction.

\subsection{Montmorillonite suspensions}

The low volume fraction results for montmorillonite as function of ionic strength were discussed in a previous article [15]. We observe that in the present study (where no salt has been added) the 
electroacoustic zeta potential of montmorillonite becomes dependent on volume fraction, especially at high volume fractions (above $0.5 \%$ ). At low volume fraction, the zeta potential is around $-60 \mathrm{mV}$ and it is about $-40 \mathrm{mV}$ at higher volume fraction for electroacoustic measurements. The low-frequency zeta potentials measured by electrophoresis (ZetaNano) are here plotted according to the formula of Smoluchowski. We have found that when fitting this low volume fraction data with both a zeta potential and a Stern layer conductance, the fitted zeta potential is closer to $-60 \mathrm{mV}$ than it appears now. Rasmusson et al. measured the electroacoustic mobility as a function of ionic strength. They found, at their lower ionic strength $(2.5 \mathrm{mM} \mathrm{NaCl})$, for a volume fraction of $0.8 \%$, a much higher zeta potential $(-105 \mathrm{mV})$ than we did at the same ionic strength, but in the range $0.025 \%-0.1 \%$ as discussed in [15]. The authors state that at the lowest AcoustoSizer measurement frequency (0.3 MHz), using the Smoluchowski formula, they find zeta potentials between -37 and $-40 \mathrm{mV}$, which are in agreement with our findings for $1 \%$ volume fraction at 3.3 MHz. They attribute the discrepancies between their fitted zeta potentials (-105 $\mathrm{mV}$ for the acoustic mobility and $-193 \mathrm{mV}$ for the zeta potential derived from their dielectric spectroscopy measurements at $2.5 \mathrm{mM}$ added $\mathrm{NaCl}$ ) and the Smoluchowski zeta potential at low frequencies to the limitation of the theory, which does not account for the size anisotropy of montmorillonite. Note that they need to use a Stern layer conductance to fit their data, as we do.

\section{Summary and conclusion}

We investigated the electrokinetic response of both kaolinite and montmorillonite particles. We conclude as follows:

for kaolinite

- In the range of volume fraction investigated $(4.6 \%$ - 20.8\%) both the conductivity and the dielectric increment are linearly proportional to the volume fraction, indicating that there are, in good approximation, no particle-particle interactions and that eq.(4) together with the theoretical formulation for the dipole coefficient (see text) can be applied to analyze the data.

- $\operatorname{Im}(\beta)$ curves cross at about $0.1 \mathrm{MHz}$ (same bulk conductivity for all volume fractions). Rowlands and O’Brien find similarly that their curves cross, but at different frequencies, which is in accordance with the theoretical predictions: 
a) Both the low frequency values of $\operatorname{Re}(\beta)$ and the fact that $\operatorname{Im}(\beta)$ curves as a function of frequency cross at different ionic strengths are linked to the presence of a Stern layer conductance.

b) The frequency at which two $\operatorname{Im}(\beta)$ curves (for two ionic strengths) cross is depending on the value of the Stern layer conductance. A more detailed study of the relation between the cross-over frequencies, ionic strength and volume fraction would be interesting.

We note that the characteristic frequencies associated to the characteristic length scales of the system are: $D / a_{n}{ }^{2}=6.5 \mathrm{~Hz}, D / a_{p}{ }^{2}=654 \mathrm{~Hz}$ and $D \kappa^{2}=4.7 \times 10^{6} \mathrm{~Hz}$. It is clear that in the case of kaolinite, the relaxation we observe is associated to $D \kappa^{2}$ and we were able to fit the data with the model, like we did in [15] when $\mathrm{KCl}$ was added to the system.

Ishida et al. who studied the high-frequency dielectric response of kaolinite found a relaxation frequency near $50 \mathrm{MHz}$. They claim that this is due to the relaxation of water molecules bound to the clay surface. Ishida et al. do not give the ionic strength at which they measure their data. From our previous study [15] we know that the relaxation frequency associated to $D \kappa^{2}$ is in the range from $10^{5} \mathrm{~Hz}$ to $10^{8} \mathrm{~Hz}$ between $0-5 \mathrm{mM}$ of added salt. We could therefore argue that the parameter we call "Stern layer conductance” could be linked (at least in some cases) to this bound water molecules relaxation. In this case, one part of the Stern layer conductance would be frequency-dependent, with a relaxation frequency around $50 \mathrm{MHz}$. Of course, more work is required to test this hypothesis.

for montmorillonite

- In the range of volume fraction investigated $(0.01 \%-0.5 \%)$ the dielectric permittivity is not linearly proportional to the volume fraction, indicating that there are particle-particle interactions, even at low volume fractions. The interactions are probably due to the long-range interactions between the faces (negatively charged) and edges (slightly positively charged) in the pH-range (around 6) where the experiments are done.

- Despite the interactions, the volume fractions used in this study, as well as in the preceding study [15], insure that the dipole coefficient extrapolated from the dielectric spectroscopy data is in good approximation constant as function of volume fraction. It remains to be investigated if that would be the case for higher volume fractions, like the ones used by Rassmusson et al. From our acoustic mobility 
data we note that around the volume fraction they used in their dielectric spectroscopy measurements (2 and $4 \%$ volume fractions) the acoustic zeta potential is extremely dependent on volume fraction. This would suggest that the dielectric spectroscopy data, and the derived dipolar coefficient are likewise volume-fraction dependent in this volume fraction range.

- In the range $10 \mathrm{kHz}-10 \mathrm{MHz}$ that we studied, we found a relaxation frequency around $8 \times 10^{4} \mathrm{~Hz}$. The characteristic frequencies associated to the characteristic length scales of the system are: $D / a_{n}^{2}=$ $1.45 \times 10^{3} \mathrm{~Hz}, D / a_{p}{ }^{2}=1.45 \times 10^{7} \mathrm{~Hz}$ and $D \kappa^{2}=1.42 \times 10^{4} \mathrm{~Hz}$. As for kaolinite, the experimental relaxation frequency is similar to the value of $D \kappa^{2}$ and the experimental dipole coefficients are in good agreement with the theoretical predictions.

Ishida et al. find a relaxation frequency at $100 \mathrm{MHz}$, and that in their case $D / a_{p}{ }^{2}=50 \mathrm{MHz}$. It would therefore appear that in the case of montmorillonite, $a_{p}$ is influencing the relaxation frequency. This is also the conclusion of Rasmusson et al. to interpret their dynamic mobility responses. A high-frequency electrokinetic study of our samples would confirm the influence of $a_{p}$ on our system.

\section{Acknowledgments}

We thank warmly the Deltares (Rotterdamseweg, Delft) for the use of their facilities. We would also like to express our deep gratitude to Jacques van der Ploeg for measuring the dielectric response of kaolinite. We also like to thank very much Matthieu Siebentritt and Grégory Lefèvre for allowing us to perform the electroacoustic measurements at the ENSCP (Paris). This investigation is financially supported by KAKEN(241583) from Japan Society for the Promotion of Science.

\section{References}

[1] N. Lockhart, "Electrical Properties and the Surface Characteristics and Structure of Clays II. Kaolinite A nonswelling Clay,” J. Colloid Interface Sci., 74, 520-529, (1980).

[2] N. Lockhart, "Electrical Properties and the Surface Characteristics and Structure of Clays I. Swelling Clay,” J. Colloid Interface Sci., 74, 509-519, (1980).

[3] W. Rowlands, R. O’Brien, “The dynamic mobility and dielectric response of kaolinite particles,” $J$. Colloid Interface Sci. 175, 190-200, (1995). 
[4] R. O’Brien, W. Rowlands, “Measuring the Surface Conductance of Kaolinite Particles,” J. Colloid Interface Sci., 159, 471-476, (1993).

[5] M. Rasmusson, W. Rowlands, R. O'Brien, R. Hunter, “The dynamic mobility and dielectric response of sodium bentonite,” J. Colloid Interface Sci., 189, 92-100, (1997).

[6] R. Raythatha, P. Sen, “Dielectric Properties of Clay Suspensions in MHz to GHz Range,” J. Colloid Interface Sci., 109, 301-309, (1986).

[7] T. Ishida, T. Makino, C. Wang, "Effects of pH on Dielectric Relaxation of Montmorillonite, Allophane, and Imogolite Suspensions.,” J. Colloid Interface Sci., 212, 152-161, (1999).

[8] T. Ishida, M. Kawase, K. Yagi, J. Yamakawa, K. Fukada, "Effects of the counterion on dielectric spectroscopy of a montmorillonite suspension over the frequency range 105-1010 Hz,” J. Colloid Interface Sci., 268, 121-126, (2003).

[9] T. Ishida, T. Makino, C. Wang, "Dielectrical-relaxation spectroscopy of kaolinite, montmorillonite, allophane and imogolite under moist conditions,” Clays and Clay Minerals, 48, 75-84, (2000).

[10] H. Van Olphen, "Surface conductance of various ion forms of bentonite in water and the electrical double layer,” The Journal of Physical Chemistry, 61, 1276-1280, (1957).

[11] R. Weiler, J. Chaussidon, “Surface conductivity and dielectrical properties of montmorillonite gels,” Clays and Clay Minerals, 16, 147-155, (1968).

[12] L. Shainberg, R. Levy, “Electrical conductivity of Na-montmorillonite suspensions,” Clays and Clay Minerals, 23, 205-210, (1975).

[13] R. Calvet, “Dielectric Properties of Montmorillonites Saturated by Bivalent Cations,” Clays and Clay Minerals, 23, 257-265, (1975).

[14] A. T. Lima, J. P. G. Loch, P. J. Kleingeld, "Bentonite electrical conductivity: a model based on series-parallel transport,” J. Appl. Electrochemistry, 40, 1061-1068, (2010).

[15] Y. Tsujimoto, C. Chassagne, Y. Adachi, "Dielectric and electrophoretic response of Montmorillonite particles as function of ionic strength.”, J. Colloid Interface Sci., in press, (2013), http://dx.doi.org/10.1016/j.jcis.2013.03.033

[16] F. Mietta, C. Chassagne, J. Winterwerp, "Shear-induced flocculation of a suspension of kaolinite as a function of pH and salt concentration,” J. Colloid Interface Sci., 336, 134-141, (2009).

[17] J. Van der Ploeg, M. Mandel, “A low frequency relative permittivity meter for electrically conducting Iiquids,” Meas. Sci. Tech., 389, 389-395, (1999).

[18] C. Chassagne, D. Bedeaux, J. P. M. v.d. Ploeg, G. J. Koper, "Polarization between concentric cylindrical electrodes," Physica A, 326, 129-140, (2003).

[19] A. S. Dukhin, V. N. Shilov, H. Ohshima, P. J. Goetz, "Electroacoustic Phenomena in Concentrated Dispersions: New Theory and CVI Experiment,” Langmuir, 15, 6692-6706, (1999).

[20] C. Chassagne, F. Mietta, J. Winterwerp, “Electrokinetic study of kaolinite suspensions,” J. Colloid Interface Sci., 336, 352-359, (2009).

[21] Y. Adachi, K. Nakaishi, M. Tamaki, "Viscosity of a Dilute Suspension of Sodium Montmorillonite in a Electrostatically Stable Condition,” J. Colloid Interface Sci., 105, 100-105, (1998).

[22] Y. Tsujimoto, M. Kobayashi, Y. Adachi, "Viscosity of dilute Na-montmorillonite suspensions in electrostatically stable condition under low shear stress,” Colloid and Surface A., in press 
[23] F. Arroyo, F. Carrique, M. Jiménez-Olivares, A. Delgado, "Rheological and Electrokinetic Properties of Sodium Montmorillonite Suspensions.,” J. Colloid Interface Sci., 229, 118-122, (2000). 


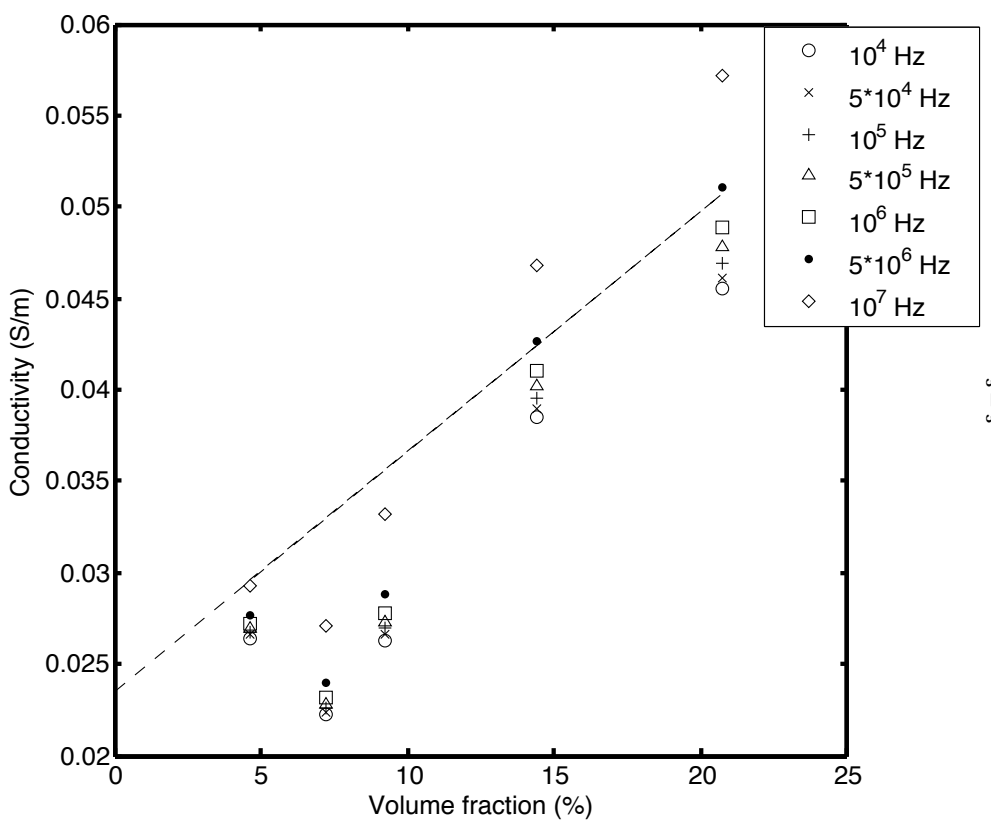

(a)

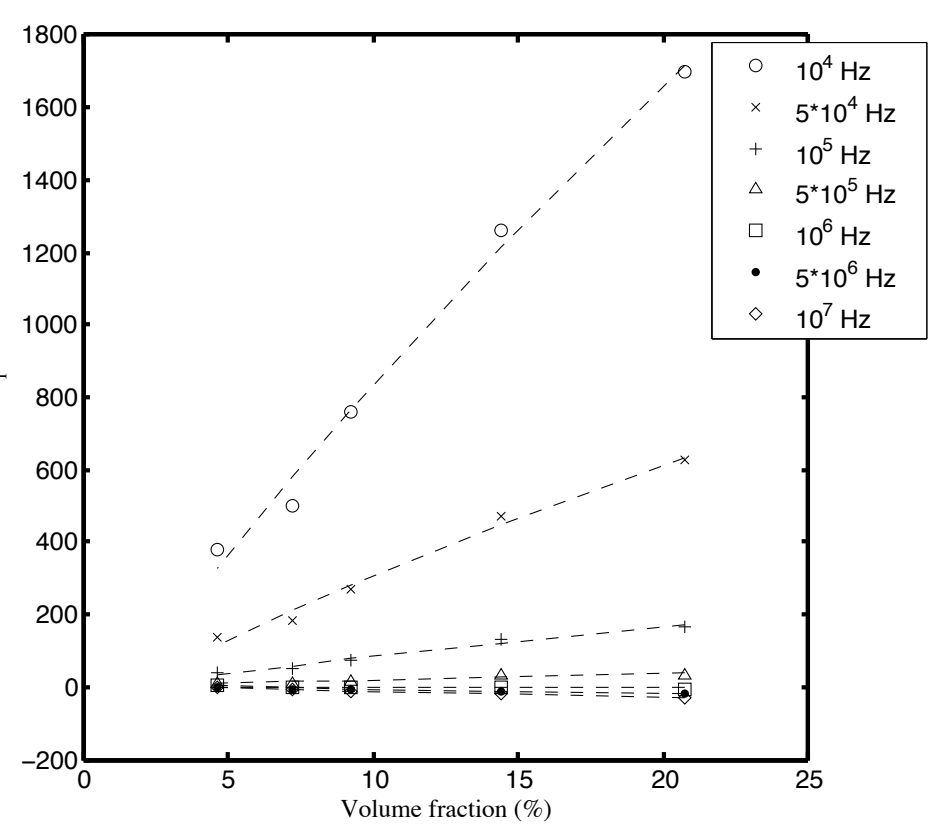

(b)

Fig.1The conductivity(a) and the dielectric permittivity (b) against volume fraction for kaolinite suspensions 

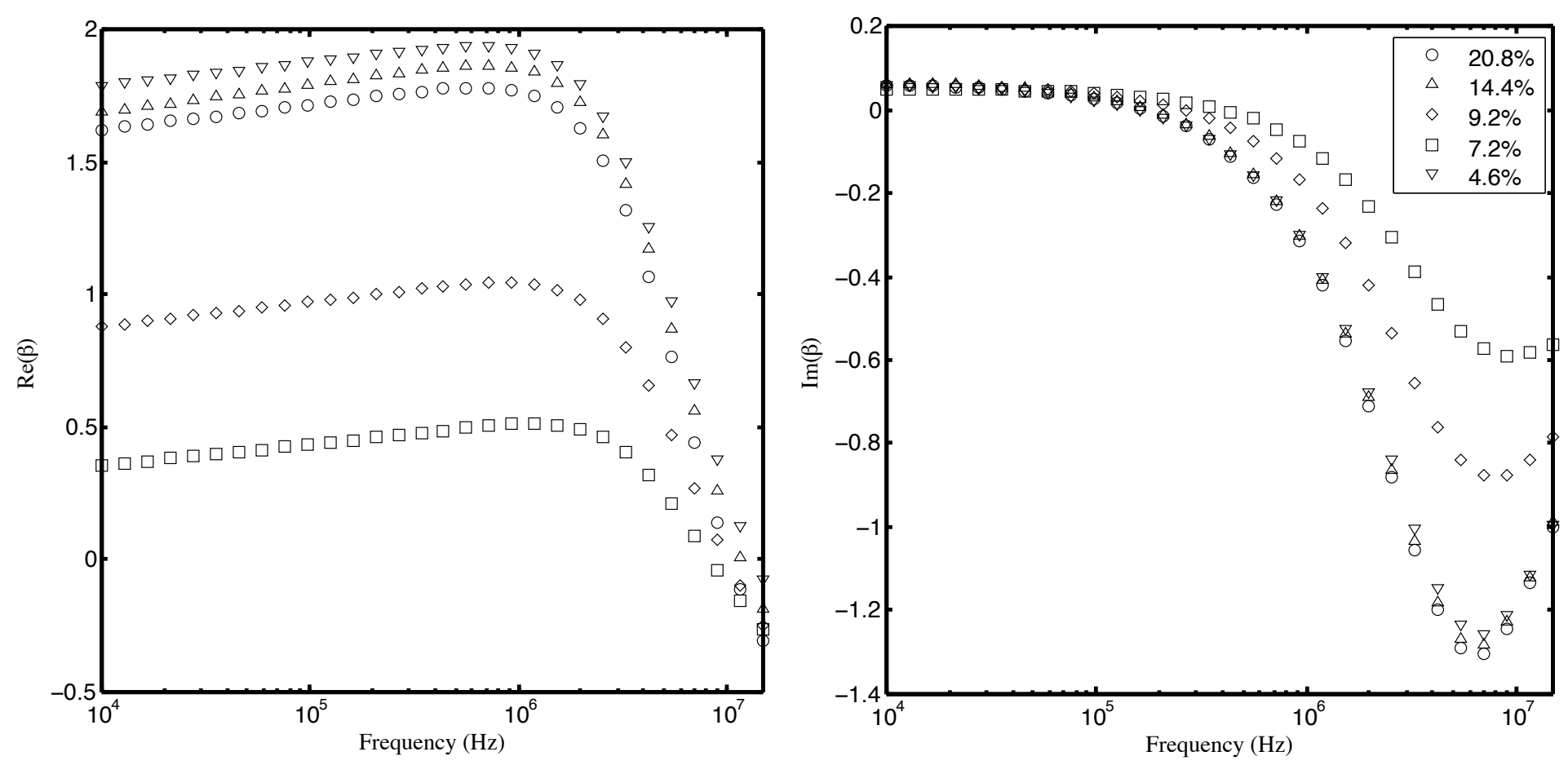

Fig.2 The experimental real and imaginary parts of the dipole coefficients of kaolinite particles for various volume fractions 

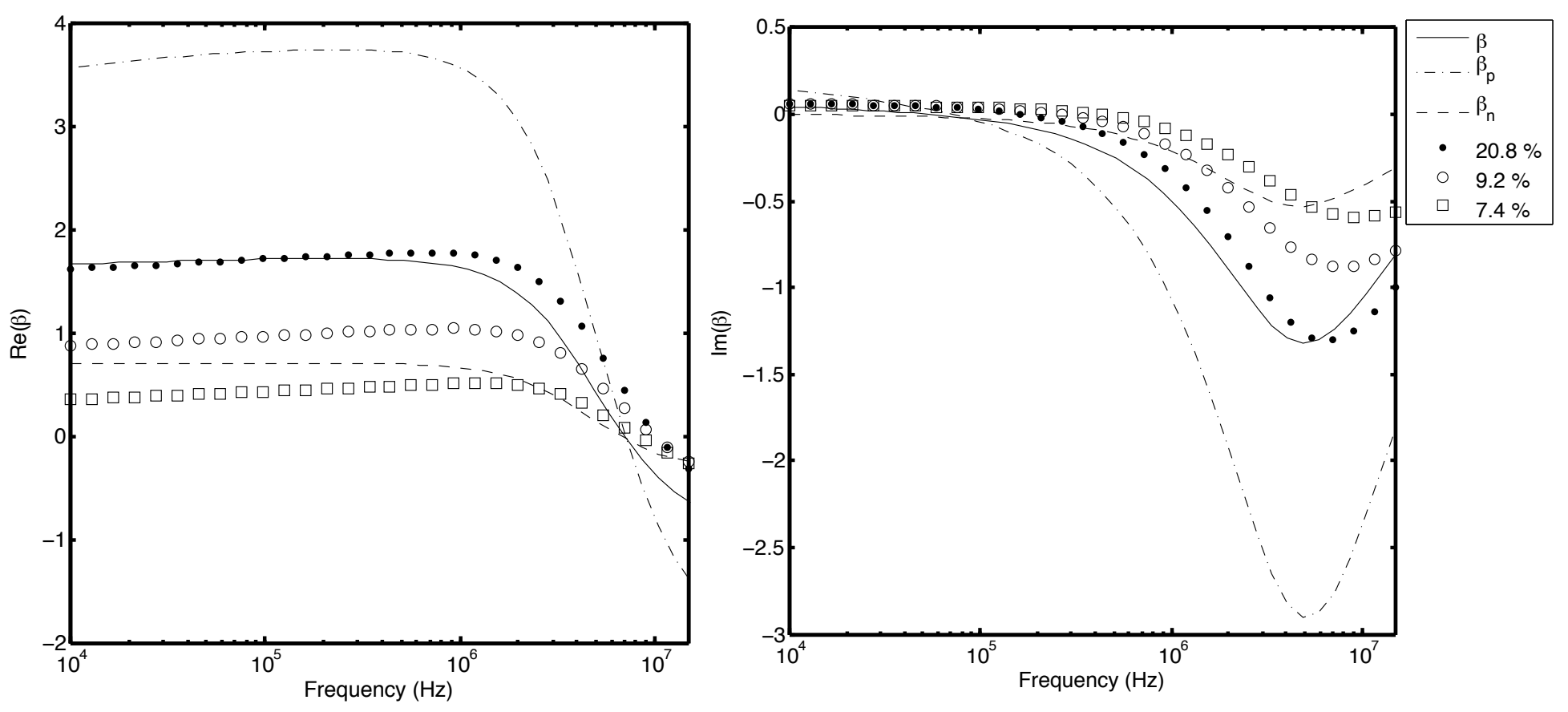

Fig. 3 the theoretical real and imaginary parts of the dipole coefficients of kaolinite particles: line:theory $(|\zeta|=75 \mathrm{mV}$, ionic strength $=1.4 \mathrm{mM}$ and $\left.K^{\text {st }}=0.0205\right)$, symbols: experimental data for different volume fraction 


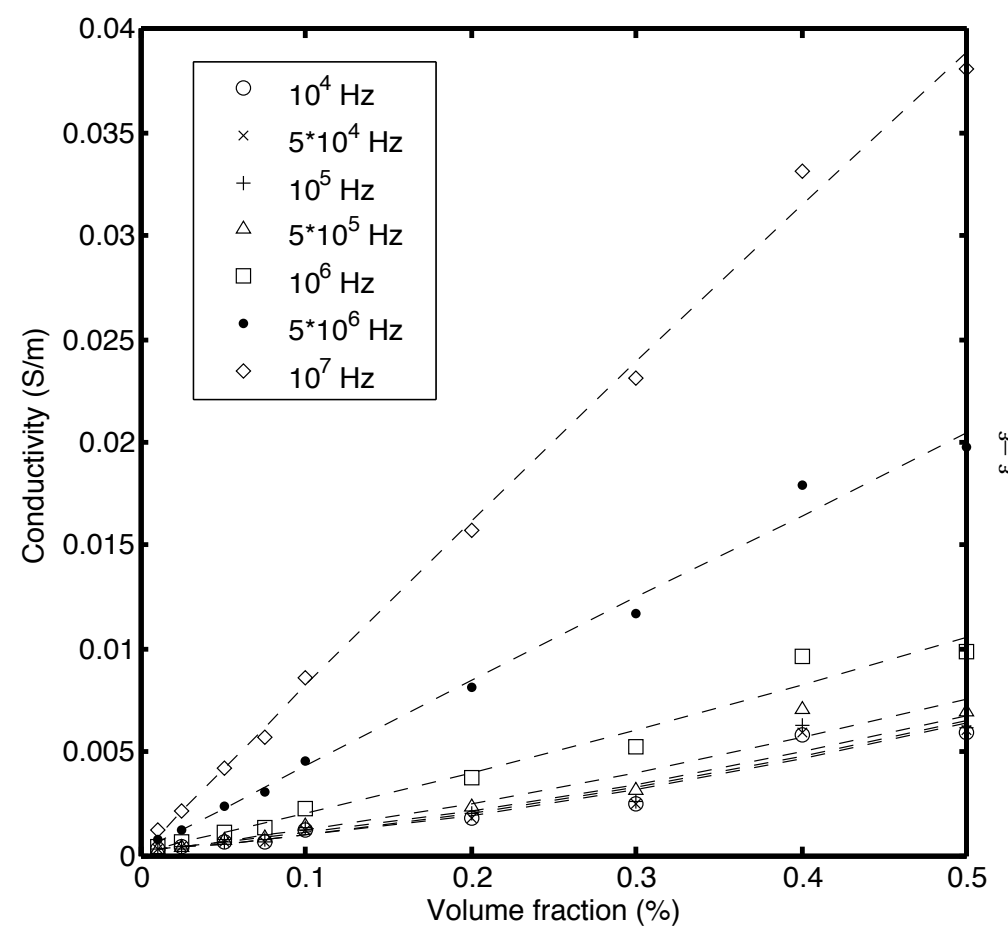

(a)

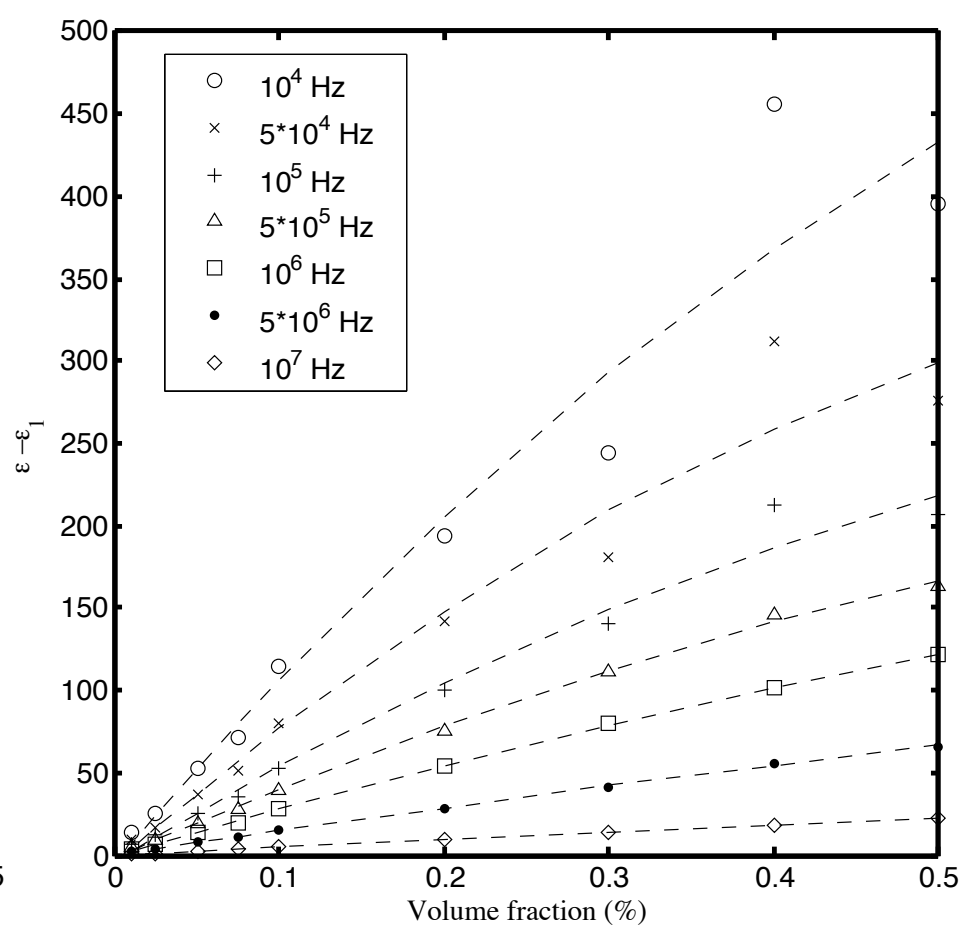

(b)

Fig.4 The conductivity (a) and the dielectric permittivity (b) against volume fraction for montmorillonite suspensions 

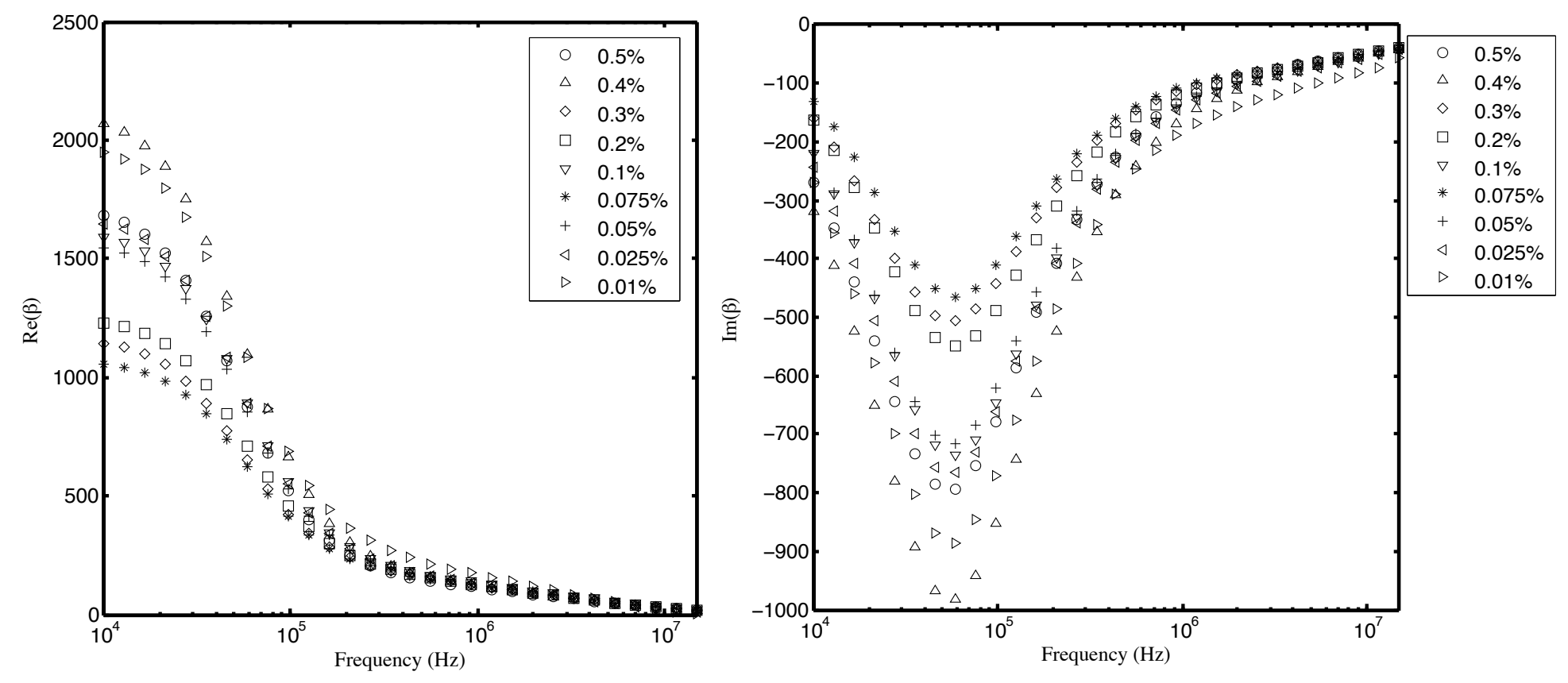

Fig.5 The experimental real and imaginary parts of the dipole coefficients of montmorillonite particles for various volume fractions 

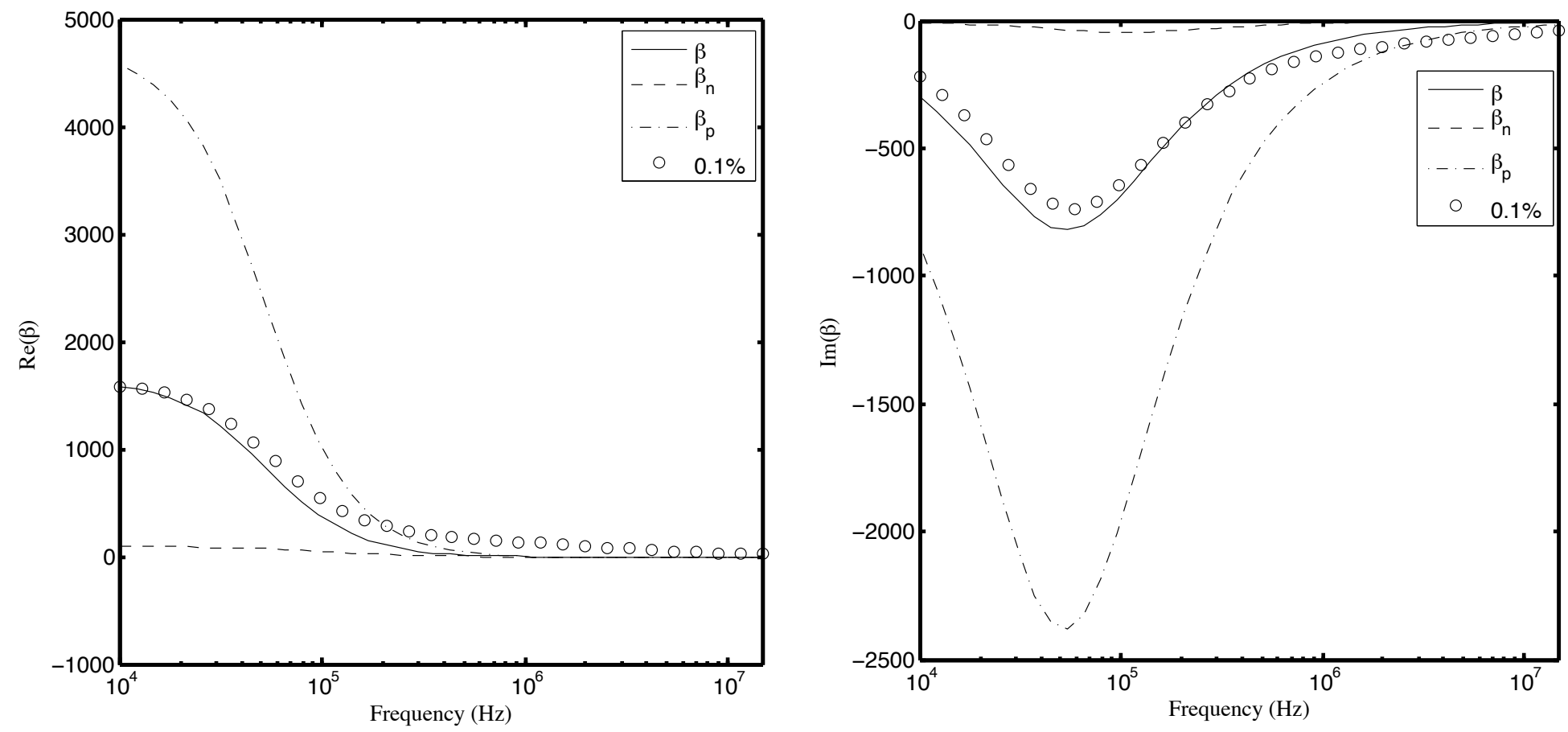

Fig. 6 the theoretical real and imaginary parts of the dipole coefficients of montmorillonite particles: lime: theory $(|\zeta|=$ $70 \mathrm{mV}$, ionic strength $=0.032 \mathrm{mM}$ and $\left.K^{s t}=0.044\right)$, symbols: experimental data for different volume farctions 


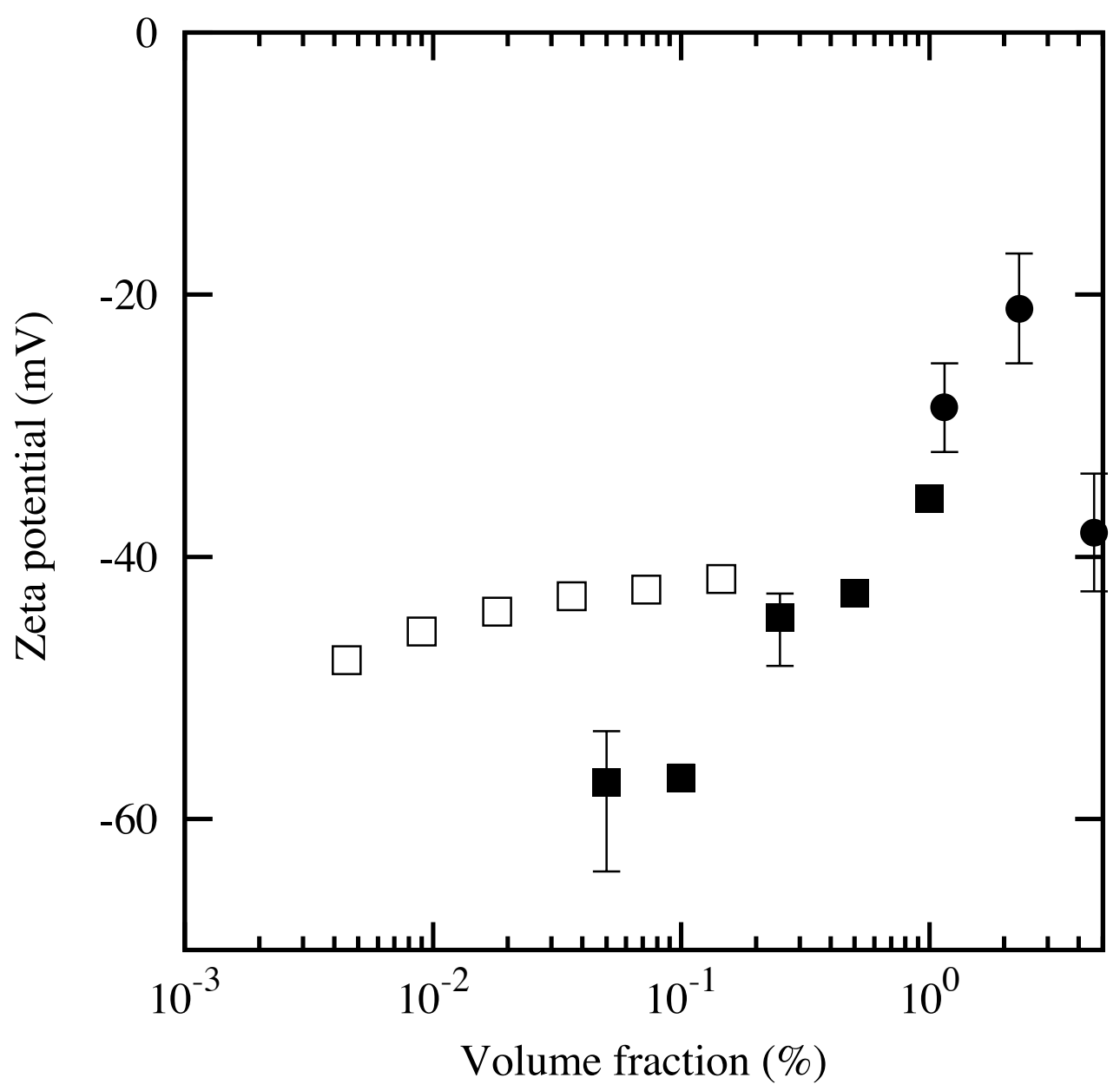

Fig. 7 the zeta potential (for montmorillonite $\square$ : Zeta-Nano, -:electro acoustic, and for kaolinite $\bullet$ : electro acoustic) as a function of volume fraction 